\title{
Radio Resource Management in NB-IoT Systems: Empowered by Interference Prediction and Flexible Duplexing
}

\author{
Hassan Malik, Muhammad Mahtab Alam, Haris Pervaiz, Yannick Le Moullec, Anwer Al-Dulaimi, Sven Pärand \\ and Luca Reggiani
}

\begin{abstract}
Narrowband Internet of Things (NB-IoT) is a promising cellular technology for enabling low cost, low power, long-range connectivity to the IoT devices. With the bandwidth requirement of $180 \mathrm{kHz}$, it provides the flexibility to deploy within the existing Long Term Evolution (LTE) band. However, this raises serious concerns about the performance of the technology due to severe interference from multi-tier 5G heterogeneous networks (HetNets). Furthermore, as NB-IoT is based on halfduplex frequency division duplexing (HD-FDD), the symmetric allocation of spectrum band between downlink and uplink results in underutilization of resource, particularly in case of asymmetric traffic distribution. Therefore, an innovative radio resource management (RRM) strategy needs to be devised to improve the spectrum efficiency and device connectivity. This article presents the detailed design challenges that need to be addressed for the RRM of NB-IoT and proposes a novel framework to devise an efficient resource allocation scheme by exploiting cooperative interference prediction and flexible duplexing techniques.
\end{abstract}

Index Terms-NB-IoT, predictive interference, duplexing techniques, radio resource management.

\section{INTRODUCTION}

$\mathbf{N}$ Arrowband-IoT (NB-IoT) is an LTE based cellular technology with a system bandwidth of $180 \mathrm{kHz}$ for supporting massive device connectivity with low-power and low-cost for delay tolerant Internet of Thing (IoT) applications. The limited bandwidth requirement of NB-IoT enables the deployment of this technology in three possible modes i.e., stand-alone, in-band and guard-band. For a stand-alone deployment, NB-IoT can operate as a dedicated carrier on a GSM channel of $200 \mathrm{kHz}$. However, for in-band and guard-band deployments, NB-IoT operates on one physical resource block (PRB) within LTE transmission. The in-band deployment mode would be more advantageous as it uses the already existing infrastructure and can be deployed as a firmware upgrade rather than deploying new hardware. Besides the basic LTE structure, some new features have been introduced in NB-IoT in order to meet the IoT requirements

Hassan Malik, Muhammad Mahtab Alam and Yannick Le Moullec are with Thomas Johann Seebeck Department of Electronics, School of Information Technologies, Tallinn University of Technology, Estonia. Emails: \{hassan.malik, muhammad.alam, yannick.lemoullec\}@taltech.ee.

Haris Pervaiz is with School of Computing and Communications (SCC), Lancaster University, UK. Emails:h.b.pervaiz@lancaster.ac.uk.

Sven Pärand is with Telia Estonia Ltd. Emails: sven.parand@telia.ee

Anwar Al-Dulaimi is with the Center of Excellence at EXFO Inc., Toronto, Canada Email:anwer.al-dulaimi@exfo.com

Luca Reggiani is with the Dipartimento di Elettronica, Informazione e Bioingegneria, Politecnico di Milano,Italy. E-mail: luca.reggiani@polimi.it and ensure the best coexistence with the LTE system. As most of the LTE control channels were designed to span over multiple PRBs using larger bandwidth than that of NB-IoT, the synchronization sequences, random access procedure and the control channel structures have been modified. Other changes include a time offset between control and data transmission for reducing complexity, the use of the lowest modulation and coding scheme (i.e., maximum allowed Quadrature Phase Shift Keying (QPSK)), retransmissions for extended coverage, etc. More details on the insight of these design changes for NB-IoT can be found in the standard [1].

Besides the advantages of NB-IoT, some issues still need to be investigated. One of the key issues is: how to efficiently use the meagre available spectrum resource (i.e., one PRB) for massive user connectivity in NB-IoT systems? As NBIoT is expected to operate in the same frequency band of LTE, this raises several design challenges for the allocation of radio resource to the IoT devices, especially in the future $5 \mathrm{G}$ Heterogenous networks (HetNets) comprising multiple smallcells operating under the umbrella of macro-cells. In this article, we describe the most critical design challenges for the radio resource management (RRM) functionalities of NB-IoT, as well as the envisioned solutions in order to describe how the different RRM functionalities should work for addressing the ambitious connectivity of IoT applications. The scope of this article is further extended to provide a novel approach for some of the issues of RRM design (i.e., inter-cell interference and asymmetric traffic between downlink and uplink) using recently introduced solutions as cooperative interference prediction (CIP) and flexible duplexing techniques.

The rest of the article is structured as follows: Sect. II includes the open research challenges for the RRM of NBIoT systems and the associated state-of-the-art solutions. Sect. III highlights the CIP and flexible duplexing techniques and describes the proposed radio resource allocation framework for NB-IoT. The detailed performance evaluation with the simulation assumptions are described in Sect. IV. Finally, the conclusions are presented in Sect. V.

\section{RESEARCH CHALLENGES FOR RADIO RESOURCE MANAGEMENT FOR NB-IOT}

This section presents some of the core issues of the RRM in NB-IoT that should be addressed in order to enable massive device connectivity. In NB-IoT, the spectrum allocation is limited to $180 \mathrm{kHz}$ mapped to one LTE PRB. As NB-IoT operates 
within the LTE band, in order to ensure the best coexistence, a standard sub-carrier spacing of $15 \mathrm{kHz}$ with 12 sub-carriers in the frequency domain is recommended. However, the subcarrier spacing can be reduced to $3.75 \mathrm{kHz}$ with 48 sub-carriers for achieving higher robustness. In the time domain, for 15 $\mathrm{kHz}$ sub-carrier spacing, the total frame duration is of $10 \mathrm{~ms}$, divided into sub-frames of $1 \mathrm{~ms}$. Each sub-frame comprises two time-slots of $0.5 \mathrm{~ms}$ and 7 orthogonal frequency-division multiplexing (OFDM) symbols. On the other hand, in the case of $3.75 \mathrm{kHz}$, each time-slot lasts $2 \mathrm{~ms}$ and the complete frame is composed of 5 time-slots (this set of parameters is one of the numerology options). Moreover, in NB-IoT, Narrowband Physical Broadcast Channel $(\mathrm{NPBCH})$ is always in the sub-frame 0 with $10 \mathrm{~ms}$ periodicity. Narrowband Primary Synchronization Signals (NPSS) and Narrowband Secondary Synchronization Signals (NSSS) occupy sub-frames 5 and 9 each $10 \mathrm{~ms}$ and $20 \mathrm{~ms}$, respectively. Narrowband Physical Downlink Control Channel (NPDCCH) is transmitted for each user and is encoded over one sub-frame. Coverage extension for NPDCCH is supported through retransmissions within a maximum of 2048 times. Similarly, in uplink, Narrowband physical random-access channel (NPRACH), used to connect users to the BS, requires a contiguous set of $12,24,36$ or 48 sub-carriers, located on a discrete set of sub-carrier ranges. For the sake of simplicity, this paper assumes $30 \%$ of the resources assigned for NPRACH [2]. The detailed design changes and features in NB-IoT that affect the spectrum efficiency are the following:

\section{A. Retransmission $\rightarrow$ to provide extended coverage and im- prove link reliability}

In NB-IoT, retransmission of data and control signals have been introduced in order to improve the coverage and system reliability in terms of packet reception. The decoding of each repeated packet can be done separately, or multiple packets can be combined for improving the rate. NB-IoT defines three coverage classes (i.e., $0 \mathrm{~dB}, 10 \mathrm{~dB}$ and $20 \mathrm{~dB}$ for Normal, Robust, and Extreme conditions, respectively) based on the signal strength, which depends on cell deployment, user distribution and propagation channel. Furthermore, with the $5 \mathrm{G}$ HetNet deployment where interference is expected to be increased due to multiple sources, the number of retransmissions also increase as the signal strength degrades. The number of retransmissions is evaluated based on the maximum coupling loss (MCL) [1]. The maximum number of retransmissions allowed in downlink and uplink are 2048 and 128, respectively.

Retransmissions improve undoubtedly the packet reception reliability but at the cost of increased latency, reduced spectrum efficiency and battery life, due to overhead and redundancy. Particularly, in NB-IoT, each retransmission adds up to 65 bytes (i.e. without header compression) or 29 bytes (i.e., with header compression). Clearly, improving the packet reception rate with retransmissions is not an optimal choice. In this regard, in [3], a 2-dimensional link adaptation considering both modulation and coding scheme (MCS) and retransmission selection for NB-IoT is presented. The proposed algorithm reduces the active time and improves spectral efficiency by providing a sub-optimal solution based on a heuristic approach. However, a more robust close-form link adaptation solution for NB-IoT is still missing. Similarly, in [4], a dynamic spectrum access for NB-IoT is proposed using reinforcement learning rather than random access. The proposed solution shows significant improvement in terms of retransmission reduction; however, the evaluation is still based on a single user scenario. One of the possible solution for reducing the retransmissions is to improve the channel quality, which is typically degraded because of intra-cell and inter-cell interferences.

\section{B. Scheduling Delay $\rightarrow$ to provide reduced computational complexity for inexpensive devices}

NB-IoT introduces a scheduling delay between the transmissions in order to reduce the computational complexity at the user devices. The NPDCCH contains the scheduling index that allows the device to receive the downlink transmission in Narrowband Physical Downlink Shared Channel (NPDSCH); it starts uplink data transmission in Narrowband Physical Uplink Shared Channel (NPUSCH) with NPUSCH Format 1 and downlink data acknowledgement (ACK) with NPSUCH Format 2. The recommended minimum scheduling delay thresholds set by 3rd Generation Partnership Project (3GPP) are shown in Fig. 1.

In downlink, the scheduling delay between NPDCCH and the associated NPDSCH is recommended to be at least $4 \mathrm{~ms}$. After the reception of NPDSCH, the user sends the associated ACK using NPSUCH Format 2 after $12 \mathrm{~ms}$ or more. The reason for such a large delay is to provide ample time for the user to decode and process the NPDSCH consisting of 680 bits compared to 23 bits of NPDCCH. Similarly, in uplink, the scheduling delay between the NPDCCH and the associated NPUSCH should be at least $8 \mathrm{~ms}$. After the completion of NPUSCH, the user needs to monitor the NPDCCH channel for at least $3 \mathrm{~ms}$ in order to receive the ACK before doing retransmission.

The scheduling delay couples the downlink and uplink transmission in NB-IoT, thus requiring joint optimisation of the resources. Furthermore, even with the best selection of the scheduling delay, there is still a significant resource wastage due to signalling overhead [6]. To address this issue, the authors in [6] proposed a pre-divided resource allocation that uses a dynamic data slicing technique for splitting the user

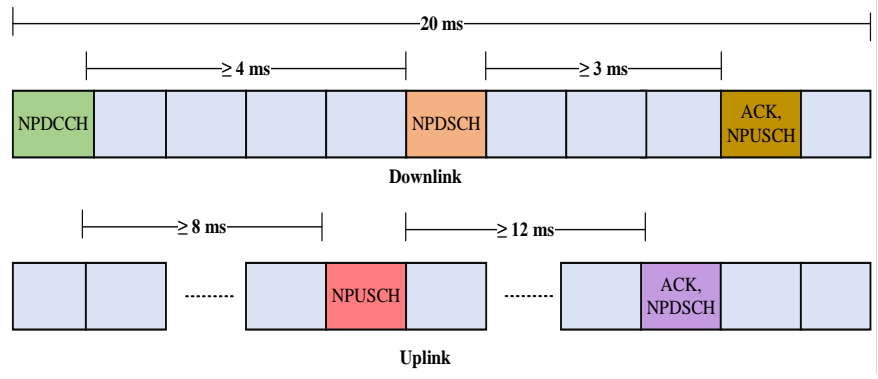

Fig. 1. Recommended scheduling delay between downlink control and data transmissions by $3 \mathrm{GPP}$ with frame duration of $20 \mathrm{~ms}$ [5]. 
resource requirements and fitting in the scattered available resources. However, it could incur excessive signal overhead since each sliced data packet requires additional PHY and MAC headers. Therefore, an appropriate scheduling mechanism for the varying payload sizes and scheduling delay selection is a challenging task, particularly for downlink transmission and this needs additional investigation.

\section{1-sub-carrier Granularity $\rightarrow$ to provide massive device support}

NB-IoT introduces the concept of resource unit (RU) for the allocation of uplink resources. The RU is defined as the combination of a number of sub-carriers (i.e., tones, the term used in the standard) and a number of symbols grouped together. Unlike traditional LTE, where the smallest allocation unit for uplink is one PRB, in NB-IoT there is a provision to allocate resources with 1-sub-carrier granularity. Such allocation provides more degrees of freedom to the base station (BS) for scheduling each uplink user over one available subcarrier (i.e., a single tone) or a group of sub-carriers (i.e., multi-tone) without limitations. For the sake of simplicity, 3GPP has already recommended pre-defined resource units for both $15 \mathrm{kHz}$ and $3.75 \mathrm{kHz}$ numerologies. The sets of RUs for NPSUCH Format 1 in $15 \mathrm{kHz}$ numerology are $1 \mathrm{~ms}$ for 12 tones, $2 \mathrm{~ms}$ for 6 tones, $4 \mathrm{~ms}$ for 3 tones, $8 \mathrm{~ms}$ for a single tone, whereas, for $3.75 \mathrm{kHz}$ numerology, only a single tone is available for $32 \mathrm{~ms}$. Furthermore, for NPSUCH Format 2, the recommended single tone allocations are $2 \mathrm{~ms}$ and $8 \mathrm{~ms}$ for $15 \mathrm{kHz}$ and $3.75 \mathrm{kHz}$ numerologies, respectively.

The allocation of uplink resources with sub-carrier granularity, however, leads to many scenarios where the uplink transmission wholly or partially overlaps in the frequency and time domains with the transmission in the neighboring cell, resulting in severe inter-cell interference (ICI). As NBIoT operates in the same LTE frequency band, this results in ICI from the neighboring cell NB-IoT users (in case of synchronous allocation of a band for NB-IoT in the neighboring cell) or from LTE users (in case of asynchronous allocation) in both downlink and uplink. Moreover, with the 5G HetNet architecture, multiple small cells operate in the vicinity of a macro-cell on the same frequency band, which adds additional ICI from the macro-cell users as illustrated in Fig. 2. ICI deteriorates performance of both downlink and uplink, however, the impact of ICI on the uplink of NB-IoT is expected to be more severe due to the provision of scheduling uplink transmissions with 1-sub-carrier granularity as the transmission might be affected by different users in different sub-carriers.

In order to combat ICI, there has been a remarkable effort in the literature for LTE. However, the traditional ICI mitigation techniques, such as Enhanced Inter-Cell Interference Coordination (eICIC), might not be suitable for NBIoT. NB-IoT is operating on a single PRB and, therefore, resource block muting in this case for NB-IoT might result in a complete system shutdown. On the other hand, resource muting in the macro-cell also reduces spectral efficiency. Specifically, for NB-IoT, the author in [3] proposes an uplink

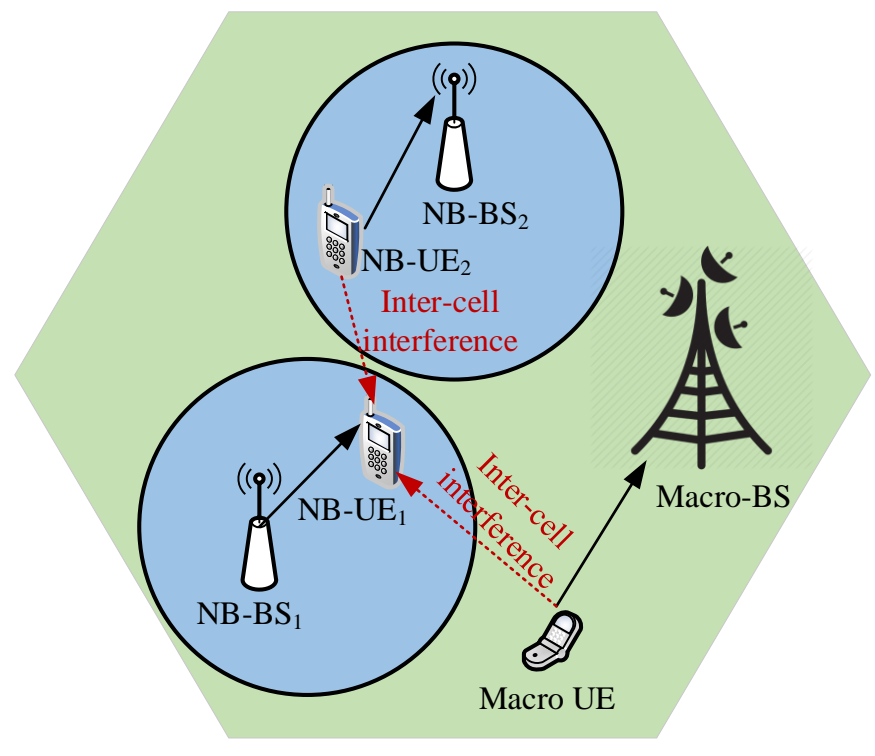

Fig. 2. Example of uplink inter-cell interference on a NB-IoT user in HetNets, where neighboring small cells have synchronous allocation of NB-IoT band.

scheduling scheme based on single-tone allocation at the linklevel without ICI consideration. Furthermore, performance of different RU configurations for NB-IoT uplink in terms of user connectivity is presented in [7]. However, the performance evaluation is conducted in a single-cell scenario and the effect of ICI, retransmission, and control channel overhead have not been considered. Moreover, in [8], the authors presented an interference-aware resource allocation based on a cooperative and non-cooperative approach for NB-IoT. It is shown that the cooperative approach improves the information rate by $11 \%$ compared to the non-cooperative one. However, the cooperative approach also adds the overhead of information sharing among the cells.

It is of utmost importance to evaluate the performance of NB-IoT in HetNets and to devise novel solutions for ICI mitigation, as traditional solutions might not be well suited to NB-IoT due to its limited bandwidth availability.

D. Half-duplex Frequency Division Duplexing (HD-FDD) support only $\rightarrow$ to reduce cost of user device and improve power efficiency

Currently, NB-IoT only provides HD-FDD support, where transmit and receive data are in a shared channel and in different timeslots, eliminating the need for an expensive duplexer. This also helps to improve the power efficiency of the user devices due to low insertion losses. However, the system with such a symmetric allocation of radio resources between downlink and uplink results in underutilization of the spectrum due to the asymmetric traffic, as in most IoT based applications the traffic between downlink and uplink is generally strongly asymmetric.

Four use-case examples with frame duration of $20 \mathrm{~ms}$, are presented in Fig. 3 with no retransmission consideration and different packet sizes in order to highlight the impact of asymmetric traffic with $15 \mathrm{kHz}$ numerology and 12 tone 


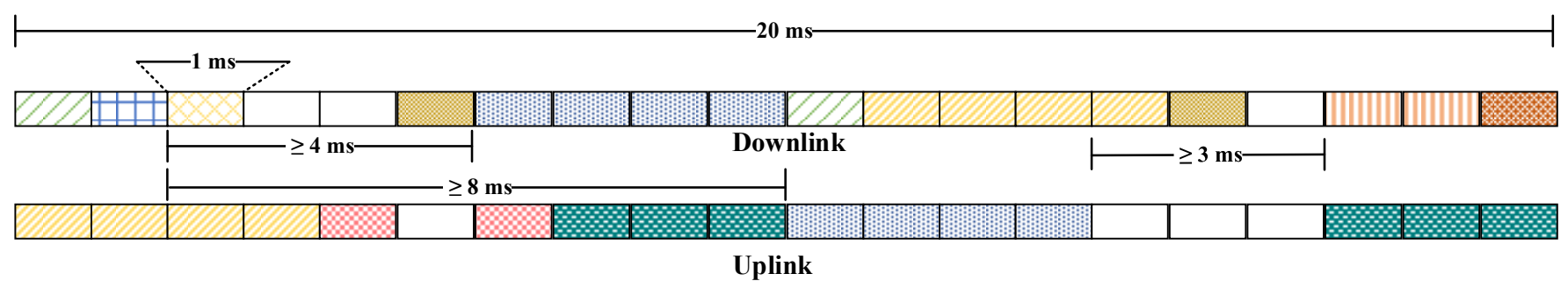

(a) Fixed payload size with resource requirement of $\{4,4,4,4,4\}$ and $\{4,4,4,4,4\}$ in downlink and uplink, respectively

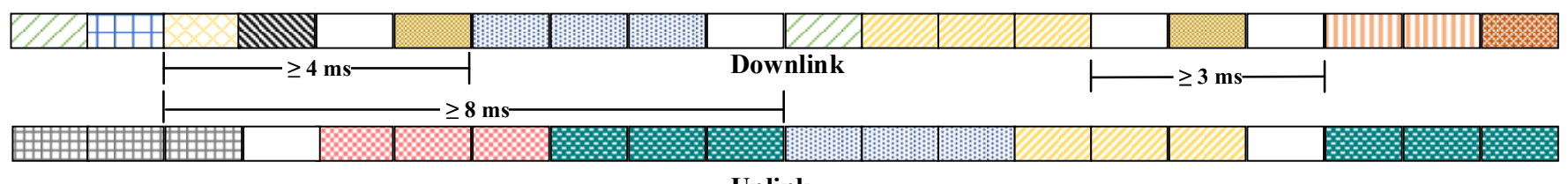

Uplink

(b) Fixed payload size with resource requirement of $\{3,3,3,3,3\}$ and $\{3,3,3,3,3\}$ in downlink and uplink, respectively

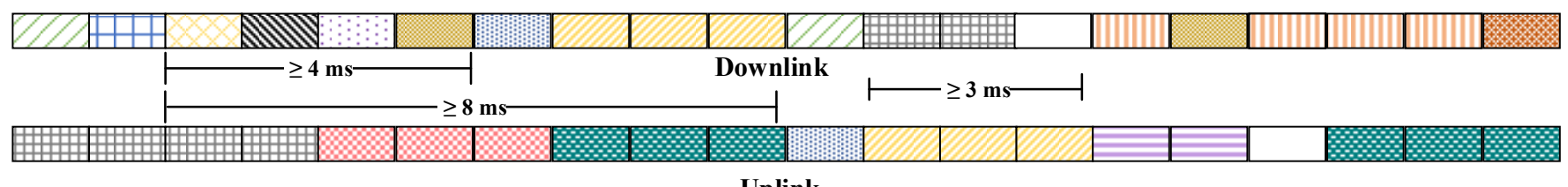

Uplink

(c) Varied payload size with resource requirement of $\{1,3,2,2,4\}$ and $\{1,3,4,2,2\}$ in downlink and uplink, respectively

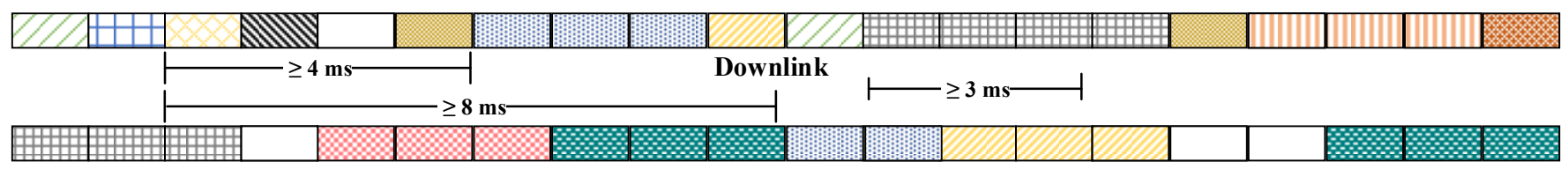

Uplink

(d) Varied payload size with resource requirement of $\{3,1,4,1,4\}$ and $\{2,3,3,1,3\}$ in downlink and uplink, respectively

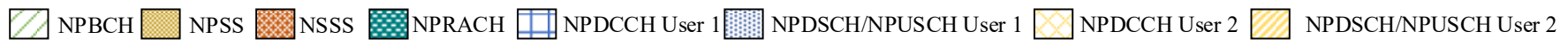
NPDSCH ACK $\square$ NPUSCH ACK NPDCCH User 3 曲 NPDSCH/NPUSCH User 3 NPDCCH User 4 N

Fig. 3. Example of downlink and uplink scheduling in NB-IoT without retransmission in four different scenarios having different RU requests for 5 users.

allocation in the uplink. Fig. 3(a) assumes five users with maximum allowed transport block size (TBS) of 1000 and 680 bits in uplink and downlink, respectively. For transmission, each user requires minimum 4 RUs and 4 sub-frames in uplink and downlink with in-band deployment due to the presence of LTE control information, as presented in [8]. Similarly, Fig. 3(b) assumes short packet size, where each user requires minimum 3 RUs and 3 sub-frames in uplink and downlink, respectively. From Fig. 3(a) it can be observed that, due to the control channel positions, there is a significant chance of resource wastage. One can surely include ACK packets in these sub-frames but, as can be seen in Fig. 3(a), only two users in the $20 \mathrm{~ms}$ frame can be accommodated. Even after including ACKs for two users, still three sub-frames in downlink and four RU's will be vacant in downlink and uplink, respectively. Similarly, in Fig. 3(b), three users can be accommodated as the packet size is shorter as compared to Fig. 3(a) scenario. However, still the resource wastage is significantly higher.

Furthermore, one might argue that, in IoT systems the traffic is mostly asymmetric in nature, therefore, the user with less RU requirements will be compensated in the vacant sub-frames as in Fig. 3(c) and Fig. 3(d). Even in these cases, there are significant chances of resource underutilization. Therefore, to fully exploit the spectrum, there is need for a joint adaptive uplink and downlink allocation with asymmetric traffic.

In traditional LTE, for a HD-FDD system, adaptive cell sizing (ACS) and multi-hop transmission (MHT) techniques were proposed to accommodate asymmetric traffic. ACS uses antenna beamforming to autonomously adjusts its coverage area resulting in uniform traffic distribution across all basestations [9]. Whereas, in MHT, a relay-station uses the uplink band to improve the downlink capacity. However, due to the deployment of NB-IoT within the LTE system, these 
techniques might not be a suitable option as they will affect the whole network architecture. One of the possible solutions is to use flexible FDD/TDD duplexing within the NB-IoT frame as it will not hamper the LTE transmission [10]. More details on flexible duplexing is presented in Section III-C.

Summing up, the RRM of NB-IoT needs to be jointly optimized for downlink and uplink to maximize the spectral efficiency and to address the issue of scheduling delay and asymmetric traffic [6], [8]. Particularly, in case of asymmetric traffic, one of the main concerns is how to utilize the vacant downlink resource for uplink. Beside this, the RRM algorithm in NB-IoT should provide an efficient interference mitigation, due to the subcarrier level of allocation, the source of interference on each subcarrier might be different as compared to LTE where the interference on whole PBR is from single source. An efficient interference mitigation not only improves the channel quality but also reduces the retransmission which is main source of spectral efficiency degradation in NB-IoT as stated in subsection II-A. Furthermore, the reduction in retransmission will also result in power and latency improvement [8].

This article addresses these challenges and presents an RRM framework by exploiting flexible duplexing and cooperative interference prediction with an objective to maximize the spectrum efficiency of NB-IoT system.

\section{Proposed Radio Resource Management FRAMEWORK}

This Section presents the proposed radio resource management framework which is constituted by three main modules, as shown in Fig. 4: CIP, resource optimisation and flexible duplexing.

\section{A. Cooperative Interference Prediction}

In NB-IoT, the BS is responsible for the allocation of resources and the selection of retransmissions and MCS to its serving users. In order to perform these functions, a BS needs to estimate the channel quality of each user in terms of signal to interference and noise ratio (SINR) using the demodulation reference symbol (DMRS). However, the estimated SINR at time $t$ can be different from the SINR during the actual transmission in $t+\Delta(\Delta$ represents time steps ahead), due to the ICI fluctuation. Assuming perfect multi-cell channel state estimation, BSs can perfectly predict ICI if they know the scheduling decisions in the neighboring cells [11]. Particularly, in case of NB-IoT, due to sub-carrier granularity, the probability of having variable interference on the sub-carrier level is significantly higher than in traditional LTE networks. Therefore, it is extremely crucial to estimate accurate ICI in advance for optimal scheduling decisions.

In this regard, CIP schemes are proposed to solve the problem [11], [12]. The core idea of CIP is to cooperate with the neighbouring $\mathrm{BS}$ and exchange the expected scheduling information of users via backhaul or X-interface before scheduling so that the interference level during the actual transmission can be predictable. For example, consider only small cells in Fig. 2; NB-BS ${ }_{2}$ estimates the scheduling probabilities of the users in advance by predicting the channel at $t+\Delta$ as in [11].
Then information is shared with $\mathrm{NB}-\mathrm{BS}_{1}$ via backhaul or $\mathrm{X}$ interface. As NB-BS ${ }_{1}$ knows which users will be scheduled at $t+\Delta$ by $\mathrm{NB}-\mathrm{BS}_{2}, \mathrm{NB}-\mathrm{BS}_{1}$ predicts the expected ICI and performs the scheduling of its users accordingly. However, since $\mathrm{NB}-\mathrm{BS}_{2}$ estimates the scheduling probabilities at time $t$, there are chances that the actual scheduling performed by NB-BS ${ }_{2}$ at at time instance $t+\Delta$ might differ as for the case in [11].

In order to improve ICI prediction accuracy, in the proposed framework, the CIP scheme presented in [11] has been modified. As in [11], the probability of scheduling is independent from previous scheduling decisions and solely relies on the predicted channel, which is justified due to the non-static position of cellular users. However, considering the static or low mobility behaviour of IoT users, our proposed scheme includes the previous scheduling decision along with the predicted channel to improve the prediction probability.

\section{B. Resource Optimisation Design}

In the resource optimisation design, with the information of user requirements and the predicted ICI $\Phi_{\text {pri }}$, the sum-rate maximization problem of BS $b$ for downlink and uplink can be formulated as $\mathrm{R}=\max _{x, p} \sum_{b \in \mathcal{B}} x \log _{2}(1+\gamma)$, where $x$ is the time-slot allocation index which indicates allocation is exclusive to one user. The user SINR $\gamma$ is given as $\frac{p \mathcal{H}}{N_{o}+\Phi}$, where $p$ represents the transmit power of the user, $\mathcal{H}$ is the channel power gain between user and serving $\mathrm{BS}, N_{o}$ is the thermal noise power and $\Phi$ is the total ICI from all neighboring cell users. The maximization problem formulation should satisfy $x \leq 1, p \leq P_{\max }, R \geq R_{\min }$ and $\Phi \leq \Phi_{\max }$, where $P_{\max }$ represents the maximum transmit power of each user, $R_{\min }$ is the minimum rate requirement of each user in both downlink and uplink, and $\Phi_{\max }$ is the maximum tolerable interference threshold by each user, respectively.

The rate maximization problems are well-known and they are non-convex due to the presence of a binary assignment variable and an interference term in the denominator [8]. Therefore, a sub-optimal solution is considered here, consisting of two steps, i.e. time-slot and power allocation, as sketched in Fig. 4.

1) Time-slot Allocation: The problem is combinatorial due to the binary variables which can be converted to a convex problem using the well-known time-sharing property [13]. With this time-sharing relaxation, maximum rate scheduling is employed by each BS for selecting the user with the highest SINR in each time-slot and thus making the best use of multi-user diversity. Each BS $b$ selects the user $i$, where $i=\arg \max _{i \in I} \gamma_{i}$; where $\mathcal{I}=\{1, \ldots, I\}$ is a set of active users in the cell.

2) Power Allocation: With the given time-slots, the power allocation problem can be simplified as follows: $\max _{p} \sum_{b \in \mathcal{B}} \log _{2}(1+\gamma)$. Due to the increase of ICI on the neighboring receivers when transmit power is increased, an interference threshold constraint is imposed so that the aggregate ICI from all the neighboring cells should be less than $\Phi_{\max }$, where $\Phi_{\max }=\Phi_{\text {pri }}$ is set to the predicted ICI. The proposed algorithm calculates first the transmit power 


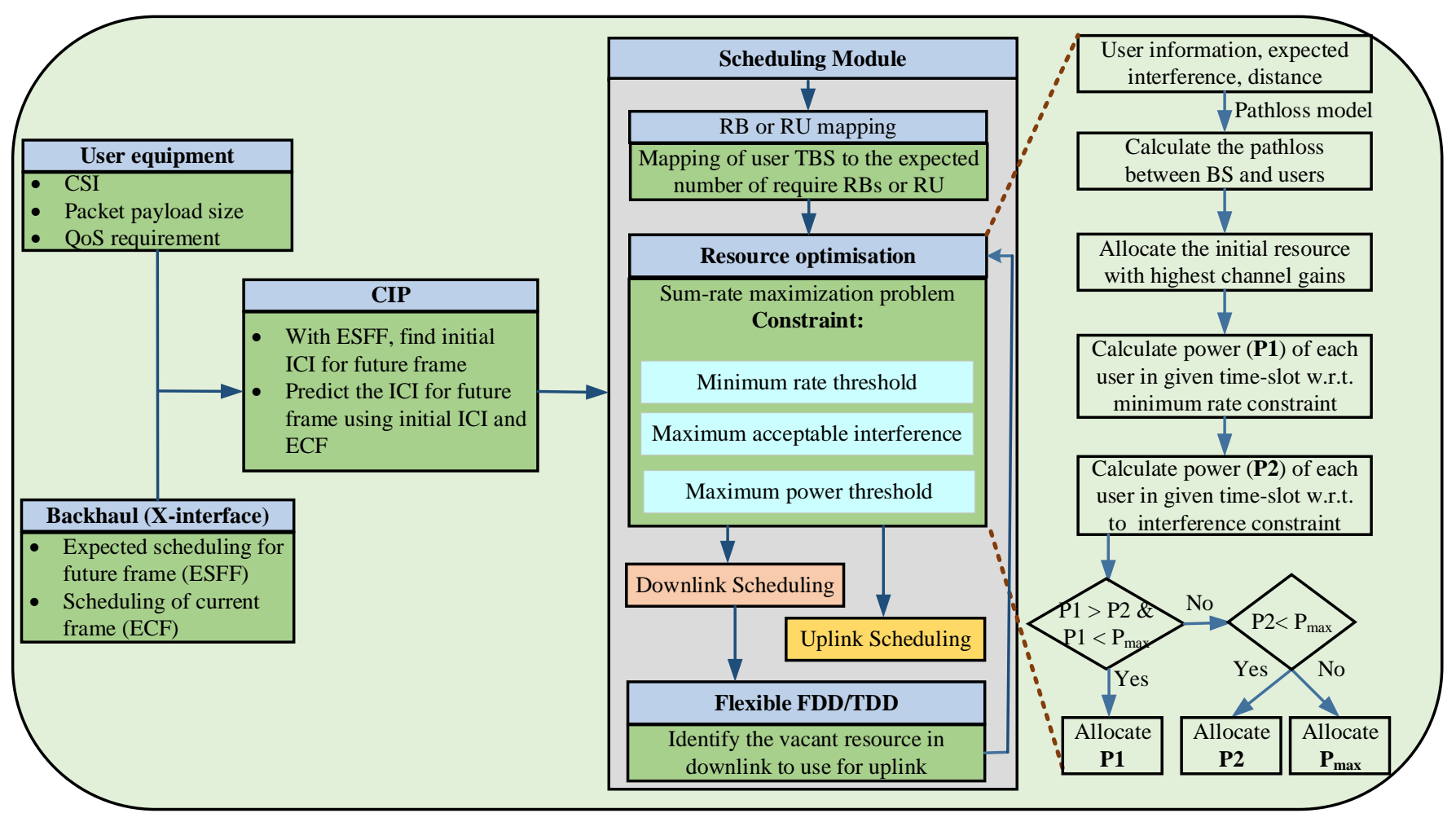

Fig. 4. Proposed radio resource management framework with cooperative interference prediction, resource optimisation and flexible duplexing module.

$P_{r}$ that satisfies the minimum rate requirement constraint, given by $\log _{2}\left(1+\frac{p \mathcal{H}}{N+\Phi}\right) \geq R_{\text {min }}$. After some mathematical manipulations, $P_{r}=\frac{1}{\mathcal{H}}\left(2^{R_{\min }}-1\right)(N+\Phi)$. As stated, the transmit power $P_{\Phi}$ is now computed based on the interference restriction imposed by the neighboring cell users in terms of predicted ICI as $P_{\Phi}=\frac{\Phi_{\max }}{\mathcal{H}}$. The final power for each user can be calculated as $P=\min \left(\max \left(P_{r}, P_{\Phi}\right), P_{\max }\right)$.

\section{Flexible FDD/TDD Duplexing}

Flexible duplexing (flexible FDD/TDD) is one of the promising technique for optimising the radio resource and coping with the channel and traffic asymmetry in wireless networks [10], [14]. The key idea is to perform adaptive resource allocation in the joint time-frequency domain, without static choices in TDD or FDD. This flexibility enables the ability to match the resources to the traffic even if there is just one paired FDD carrier, i.e. one downlink and one uplink carriers. Furthermore, the use of TDD enables to allocate resources at finer granularity, i.e. symbol level rather than carrier level for FDD.

As a result, flexible duplexing has the advantage of accommodating asymmetric traffic with better spectral efficiency. However, one of the major challenges for exploiting the maximum potential of flexible duplexing is the inter-cell interference between the downlink and uplink users. For example, if a user in a cell performs uplink transmission using downlink resources, it will receive interference from the neighbouring cell downlink users, which might be significantly higher because of the transmit power gap between downlink and uplink. In order to address this issue, in [14], the authors proposed a resource muting approach within the flexible duplexing framework. However, resource muting might not be a suitable choice for NB-IoT due to limited system bandwidth.

Our proposed radio resource framework exploits the flexible duplex and address the ICI interference from downlink user to uplink user and viceversa. In this regard, the proposed resource optimisation schedules initially the users in downlink since the downlink traffic is significantly lower than the uplink one in IoT context. Then the downlink scheduling information is used by a flexible duplexing module for calculating the SINR of each uplink user using predicted ICI from downlink users in that time-slot as follows: $\gamma_{u p}=\frac{p \mathcal{H}}{N+\Phi_{p r i}}$. Then the user with maximum SINR in that time-slot is selected to perform uplink and the corresponding power level is calculated as presented in the previous section.

\section{Performance Evaluation}

This section presents the performance evaluation of the proposed framework through Monte Carlo simulation which runs for 5000 iterations. The scenario is a multi-cell LTE network comprising three sectors and an inter-site distance equal to $500 \mathrm{~m}$ with synchronous PRB allocation for NB-IoT users in each cell, in both in-band and stand-alone modes. Furthermore, the total frame duration is assumed to be $20 \mathrm{~ms}$ with $15 \mathrm{kHz}$ numerology, where RU is made of 12 tones for $1 \mathrm{~ms}$. A total number of 20 users are deployed randomly and uniformly in each cell with a maximum transmit power of $35 \mathrm{dBm}$ and $23 \mathrm{dBm}$ in downlink and uplink, respectively. A penetration loss of $35 \mathrm{~dB}$ is assumed to ensure that high coupling losses are achieved, as they approximately lies in the 


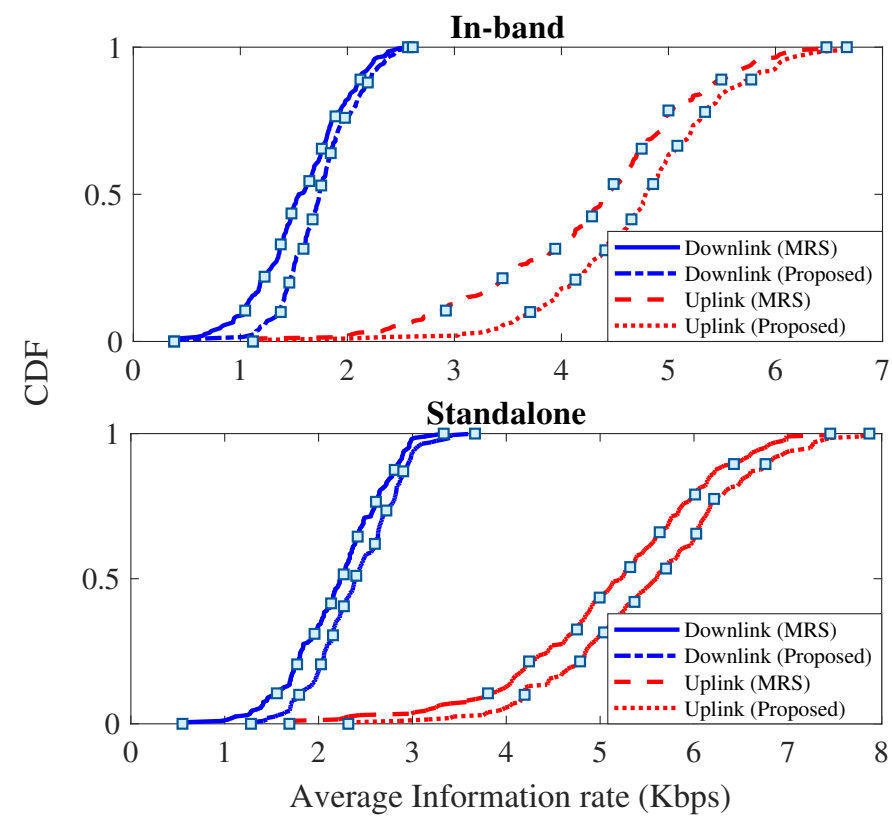

Fig. 5. Average information rate per sector with in-band and stand-alone deployment and random packet size.

range from 8 to $38 \mathrm{~dB}$. The rest of the simulation assumptions follow closely the 3GPP standard and are presented in [15].

Furthermore, considering that in most IoT applications downlink is only used for control information, the TBS for each downlink and uplink user is randomly generated between [10 20] and [20 50], respectively. In terms of MCS, each user applies QPSK and a $1 / 3$ code rate on all the transmissions. However, the retransmission factor is computed with respect to MCL for different coverage classes as given in [15].

The results are generated for the average information rate per sector, as shown in Fig. 5 through the cumulative distribution function (CDF). Due to the lack of resource allocation schemes for NB-IoT, we have compared the proposed resource allocation with the maximum rate scheduling (MRS), where

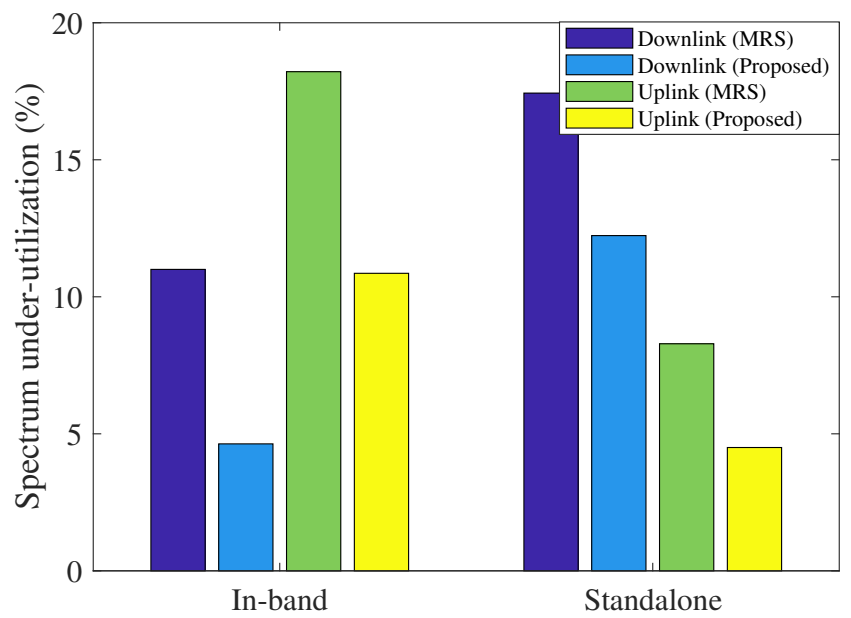

Fig. 6. Percentage of under-utilized spectrum resource per sector in both in-band and stand-alone deployments. users are allocated according to the priority given by the maximum channel gain without the interference information, in order to highlight the gain that can be achieved with CIP. It can be seen that the proposed framework significantly improves the downlink and uplink performance in in-band by approximately $11 \%$ and $14 \%$, respectively and, in the standalone mode, by $9 \%$ and $12 \%$. The reason for this improvement is the reduction in retransmissions due to interference-aware scheduling, which helps to reduce the effect of ICI and improves the link reliability. In addition, flexible duplexing enables uplink to transmit on vacant downlink resources and viceversa, which also contributes in the gain improvement in both downlink and uplink, as can be seen in Fig. 6. The results show that the proposed RRM framework is able to achieve reductions around $58 \%$ and $40 \%$ in resource wastage in downlink and uplink respectively, in in-band deployment and $30 \%$ and $45 \%$ in downlink and uplink, respectively, in the stand-alone one.

\section{Conclusions}

NB-IoT is a promising technology for enabling future IoT applications. However, scarce system bandwidth and frequency sharing with LTE raise several challenging issues that need to be addressed in order to exploit fully the potential of this technology. This paper highlighted some of the major RRM issues that need to be tackled for achieving the maximum spectral efficiency. Moreover, the paper also presented a novel RRM framework specifically designed for NB-IoT and exploiting the CIP and flexible duplexing techniques for addressing those issues. Simulation results show that the proposed framework mitigates significantly the impact of ICI, reduces the retransmissions, accommodate asymmetric traffic and improve the overall spectrum resource utilization. It is observed that with the proposed RRM framework up to $14 \%$ improvement in terms of information rate and $58 \%$ reduction in resource wastage can be achieved.

\section{ACKNOWLEDGMENT}

"This project has received funding from European Unions Horizon 2020 Research and Innovation Program under Grant 668995 and partly through ESPRC UK Global Challenges Research Fund (GCRF) allocation under Grant EP/P028764/1. This material reflects only the authors view and the EC Research Executive Agency is not responsible for any use that may be made of the information it contains."

\section{REFERENCES}

[1] "3GPP TR45.820: Cellular System Support for Ultra Low Complexity and Low Throughput Internet of Things," Tech. Rep., 2015.

[2] "3GPP R1157199: NB-IoT Initial analysis of PRACH capacity ," Tech. Rep., 2015.

[3] C. Yu, L. Yu, Y. Wu, Y. He, and Q. Lu, "Uplink Scheduling and Link Adaptation for Narrowband Internet of Things Systems," in IEEE Access, vol. 5, pp. 1724-1734, 2017.

[4] M. Chafii, F. Bader, and J. Palicot, "Enhancing coverage in narrow bandIoT using machine learning," in IEEE Wireless Communications and Networking Conference (WCNC), 2018, pp. 1-6.

[5] Y. P. E. Wang, X. Lin, A. Adhikary, A. Grovlen, Y. Sui, Y. Blankenship, J. Bergman, and H. S. Razaghi, "A Primer on 3GPP Narrowband Internet of Things," IEEE Communications Magazine, vol. 55, no. 3, pp. 117123, 2017. 
[6] R. Boisguene, S. Tseng, C. Huang, and P. Lin, "A survey on NBIoT downlink scheduling: Issues and potential solutions," in 13th International Wireless Communications and Mobile Computing Conference (IWCMC), 2017, pp. 547-551.

[7] R. C. J. Neto, E. B. Rodrigues, and C. T. Oliveira, "Performance Analysis of Resource Unit Configurations for M2M Traffic in the Narrowband-IoT System," in 35th Brazilian Communications and Signal Processing Symposium, 2017.

[8] H. Malik, H. Pervaiz, M. M. Alam, Y. Le Moullec, A. Kuusik, and M. A. Imran, "Radio Resource Management Scheme in NB-IoT Systems," in IEEE Access, vol. 6, pp. 15 051-15064, 2018.

[9] H. Malik, M. Ghoraishi, and R. Tafazolli, "Cross-layer approach for asymmetric traffic accommodation in full-duplex wireless network," in European Conference on Networks and Communications (EuCNC), 2015, pp. 265-269.

[10] K. Balachandran, J. H. Kang, K. Karakayali, and K. M. Rege, "Flexible duplex in FDD spectrum," in IEEE International Conference on Communications Workshops (ICC Workshops), 2017, pp. 296-301.

[11] F. Diehm and G. Fettweis, "Cooperative interference prediction for enhanced uplink link adaptation under backhaul delays," in IEEE 23rd International Symposium on Personal, Indoor and Mobile Radio Communications - (PIMRC), 2012, pp. 173-177.

[12] Q. Li, C. Li, Y. Wu, S. Feng, and P. Zhang, "An Investigation on Cooperative Interference Prediction in LTE-A Indoor Hotspot Scenarios," in IEEE 81st Vehicular Technology Conference (VTC Spring), 2015, pp. $1-6$.

[13] Z.-Q. Luo and W. Yu, "An introduction to convex optimization for communications and signal processing," IEEE Journal on Selected Areas in Communications, vol. 24, no. 8, pp. 1426-1438, 2006.

[14] Q. Liao, "Dynamic uplink/downlink resource management in flexible duplex-enabled wireless networks," in 2017 IEEE International Conference on Communications Workshops (ICC Workshops), 2017, pp. 625631.

[15] “3GPP R1157248: NB IoT Capacity evaluation, Nokia Networks ," Tech. Rep., 2015.

\section{BIOGRAPHIES}

Hassan Malik (M'18) received his B.E. degree from National University of Sciences and Technology (NUST), Pakistan, in 2009, M.Sc. degree from the University of Oulu, Finland, in 2012, and Ph.D. degree from 5G Innovation Centre (5GIC), University of Surrey, UK, in 2017. Hassan worked as a research assistant at the Centre for Wireless Communication (CWC), Oulu, Finland, from 2011 to 2013. He is currently working as a researcher in the Thomas Johann Seebeck Department of Electronics at Tallinn University of Technology, Estonia. His current research interests include wireless communication protocols, IoT, URLLC, LPWAN technologies, blockchain-based IoT, and embedded systems.

Muhammad Mahtab Alam (M07) received his Ph.D. degree from University of Rennes1 (INRIA Research Center), France, in 2013. He conducted postdoctoral research with Qatar Mobility Innovations Center, from 2014-2016. In 2016, he was elected as the European Research Area Chair in the Cognitive Electronics Project and an Associate Professors with Tallinn University of Technology (TalTech), Estonia. In 2018, he obtained tenure professorship under cooperation framework between Telia and TalTech. He has authored or coauthored over 60 research publications. His research interests include self-organized wireless sensor, body area networks, internet of things, public safety and critical networks, and digital signal processing.

Haris Pervaiz (S'09-M'09) received the M.Sc. degree from Royal Holloway University of London, Egham, U.K., in 2005, and the Ph.D. degree from the School of Computing and Communication (SCC), Lancaster University, U.K., in 2016. Currently, he is working as an Assistant Professor at SCC, Lancaster University, UK. From 2017 to 2018, he was a Research Fellow with the 5G Innovation Centre, University of Surrey, U.K. From 2016 to 2017, he was an EPSRC Doctoral Prize Fellow with Lancaster University. His current research interests include green heterogeneous wireless communications, 5G and beyond, millimeter wave communication, and energy and spectral efficiency.

Yannick Le Moullec received the MSc (EE) degree from Université de Rennes I (France) in 1999 and the PhD (EE) degree from Université de Bretagne Sud (France) in 2003. From 2003 to 2013 he successively held postdoc, assistant, and associate professor positions at Aalborg University (Denmark). From 2013 to 2016 is was a senior researcher at Tallinn University of Technology (Estonia) where he then got a professorship in 2017. His research interests span HW/SW co-design, embedded systems, reconfigurable systems, and IoT.

Sven Pärand received both his M.Sc. degree (2006) in telecommunications and his Ph.D. degree (2018) in electronics and telecommunication from Tallinn University of Technology, Estonia. He has been an engineer at Telia Estonia Ltd since 2012, initially working on IMS and migration towards the Next Generation Networks. Starting from 2018, he is working as a $5 \mathrm{G}$ development manager responsible for implementing the $5 \mathrm{G}$ network at Telia Estonia.

Anwer Al-Dulaimi is a technical product owner in the Center of Excellence at EXFO Inc., Montréal, Canada. His research interests include $5 \mathrm{G}$ networks, cloud computing, vehicle to everything, and the Internet of Things. $\mathrm{He}$ is IEEE Distinguished Lecturer and chair of IEEE 1932.1 standardization working group for Licensed/Unlicensed Spectrum Interoperability in Wireless Mobile Network.

Luca Reggiani, $\mathrm{PhD}$ in Electronics and Communications Engineering, is currently a researcher at Politecnico di Milano (Italy). He has collaborated with several industries and Universities in the field of wireless communications and magnetic recording, as a consultant or within Italian and European research programs. His research interests include mobile systems, wireless sensor networks, high capacity point-to-point links, information theory. 